\title{
动态磁涂覆混合纳米固定相毛细管电色谱中芳香酸的输运特征研究
}

\author{
张凌怡朱亚仙张维冰* \\ (华东理工大学 上海市功能性材料化学重点实验室 上海 200237)
}

\begin{abstract}
摘要 推导了芳香酸在动态磁涂覆混合纳米固定相开管毛细管柱中等度洗脱和二元台阶梯度洗脱条件下的输运方程, 考察了其在该色谱柱上的保留特征. 结果表明, 等度洗脱时, 电渗流方向及大小随着两种固定相配比的改变而改变, 溶质的容量因子与弱阴离子交换固定相和反相固定相比例 $\left(\mathrm{Fe}_{3} \mathrm{O}_{4} @ \mathrm{SiO}_{2}-\mathrm{NH}_{2}: \mathrm{Fe}_{3} \mathrm{O}_{4} @ \mathrm{SiO}_{2}-\mathrm{C}_{18}\right)$ 之间具有良好的线性关 系, 拟合后相关系数大于 0.9166 . 二元台阶梯度洗脱条件下, 溶质的迁移时间可以通过其在等度洗脱条件下的洗脱时 间加以预测. 溶质在经磷酸缓冲液浓度、有机调节剂浓度和 $\mathrm{pH}$ 值台阶梯度洗脱后，其预测保留时间和实际保留时间之 间的相对误差分别小于 $8.0 \%, 10 \%$ 和 5.8\%. 构建的系统在优化的色谱条件下对水提天麻进行了分离, 其主要成分柠檬 酸的保留时间预测值与实际值之间相对误差为 $11 \%$.
\end{abstract}

关键词 梯度洗脱; 输运特征; 混合固定相

\section{Transport Characteristics of Aromatic Acids in the Capillary Electrochroma- tography with Mixed Magnetic Nanoparticles Coating as Stationary Phase}

\author{
Zhang, Lingyi Zhu, Yaxian Z Zhang, Weibing* \\ (Shanghai Key Lab of Functional Materials Chemistry, East China University of Science and Technology, Shanghai 200237)
}

\begin{abstract}
In this paper, novel open-tubular electrochromatographic system with core/shell magnetic nanoparticles modified by amino and $\mathrm{C}_{18}$ functional groups as mixed stationary phase by using a series of permanent magnets to immobilize nanoparticles into the capillary were investigated. The new system showed high separation ability and efficiency due to high specific surface area of nanomaterial and mixing separation mechanism. The transport equations of aromatic acids using the open-tubular electrochromatographic column with magnetic nanoparticle coating as stationary phases were derived under isocratic and double stepwise gradient elutions, and the retention characteristics of organic acids separated by the columns were further investigated. The results showed that the electroosmotic flow gradually decreased with the increase of $\mathrm{Fe}_{3} \mathrm{O}_{4} @ \mathrm{SiO}_{2}-\mathrm{NH}_{2}$ in the mixed stationary phases, and the direction of electroosmotic flow reversed eventually. The capacity factor was relevant to the ratio of ion-exchange and reversed-phase stationary phases. Furthermore, experiments confirmed that the capacity factor of solute had a good linear relationship with the ratio of mixed stationary phases, and the correlation coefficient was greater than 0.9166 after fitting. Under the double stepwise gradient elution condition, the migration time of solute can be predicted by its elution time under isocratic elution. After the solutes were eluted by phosphate buffer stepwise gradient elution, the relative errors between predicted retention time and actual retention time of solute were less than $8.0 \%$. After they were eluted by organic modifier concentration stepwise gradient elution, the relative errors between two values of solute were less than $10 \%$. After they were eluted by $\mathrm{pH}$ value stepwise gradient elution, the relative errors between two values of solute were less than $5.8 \%$. Rhizoma gastrodiae was separated by the constructed system under the optimized chromatographic condition, the main components of gastrodin, vanilla alcohol and citric acid were identified based on retention time of standards, and the relative errors between predicted value and actual value of citric acid was $11 \%$. The open-tubular electrochromatographic system shows great potential in the method development of analysis of complicated samples.

Keywords gradient elution; transport characteristic; mixed stationary phase
\end{abstract}

\section{1 引言}

毛细管电色谱(CEC)以电渗流(EOF)驱动流动相进 行样品的分离, 因此在完成梯度洗脱过程时所采用的方 法与高效液相色谱(HPLC)不同. 在毛细管电色谱中，常 用的梯度洗脱包括线性梯度洗脱和台阶梯度洗脱等. 线
性梯度的洗脱通过改变流动相的组成 ${ }^{[1,2]}$ 、操作电压 ${ }^{[3,4]}$ 和温度 ${ }^{[5]}$ 等参数实现. 高效液相色谱中广泛采用的流动 相线性梯度洗脱在毛细管电色谱中也是一种较为理想 的梯度洗脱模式，通过改变流动相的组成使溶质的迁移 速度发生改变, 从而改变其分离特征而达到改变分离选

*E-mail: weibingzhang@ecust.edu.cn; Tel.: 021-64252145; Fax: 021-64233161

Received October 13, 2012; published December 10, 2012.

Supporting information for this article is available free of charge via the Internet at http://sioc-journal.cn.

Project supported by the National Natural Science Foundation of China (No. 21075039), the Specialized Research Fund for the Doctoral Program of Higher Education of China (No. 20100074110016) and the Fundamental Research Funds for the Central Universities (No. WK1014042).

项目受国家自然科学基金(No. 21075039)、教育部(No. 20100074110016)和中央高校基金科研业务费专项资金(No. WK1014042)资助. 
择性的目的. Sepaniak 和 Tsuda ${ }^{[6,7]}$ 采用线性混合器的方 法进行线性梯度洗脱, 他们都是通过向缓冲液储液池中 不断注入新的缓冲液从而改变流动相组成. Sepaniak ${ }^{[6]}$ 的溶剂传输系统能产生连续线性的, 凹字形和凸字形的 溶剂梯度. 他们设计了双洜体系, 其中一个泵用于向入 口处储液池注入新的缓冲液, 另一个㫤用于在出口处储 液池抽去过量的溶剂. Tsuda ${ }^{[7]}$ 的系统则通过使用一个分 流进样装置来改变入口和出口处储液池中缓冲液的组 成. 该方法除了需要使用额外的设备外, 还需要考虑溶 剂溢出、缓冲液储液池和样品储液池的切换等问题, 较

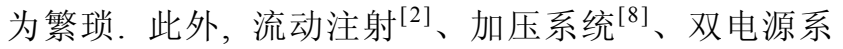
统 ${ }^{[9,10]}$ 、自动微梯度系统 ${ }^{[1,12]}$ 等方法也被用于毛细管电 色谱线性梯度洗脱.

台阶梯度是一种最简单的梯度洗脱模式, 可以在大 多数电色谱仪器上便利实现, 与线性梯度中逐渐、连续 的增加相反, 它能在预设的时间内实现缓冲液强度的瞬 间增加. 常见的台阶梯度方法包括机械台阶梯度 ${ }^{[13]}$ 、电 动门台阶梯度 ${ }^{[14,15]}$ 和采用混合器的台阶梯度 ${ }^{[16,17]}$ 等. Euerby ${ }^{[13]}$ 首先采用机械台阶梯度方法对 6 种利尿剂进行 了分离, 与常规的等度洗脱相比, 分离时间缩短了 19 $\min$, 且样品达到了完全分离, 三次二元台阶梯度洗脱 后溶质保留时间的相对误差在 $0.5 \% \sim 1.0 \%$ 之间. 张维 冰等 ${ }^{[18]}$ 从理论上研究了二元台阶梯度洗脱下电渗流和 中性溶质的迁移特征. 以 12 种芳香化合物为样品, 从理 论上预测其在 CEC 中的输运规律, 并进一步通过实验 验证. 结果表明, 溶质保留时间的理论值和实验值的相 对误差小于 $5.0 \%$, 说明推导的理论表达式能很好地描 述中性溶质在毛细管电色谱二元台阶梯度洗脱中的输 运规律. 与线性梯度相比, 台阶梯度在样品进行分离时, 缓冲溶液的操作少了一定的灵活性. 此外, 毛细管的进 样端容易引入气泡, 导致填充柱的使用寿命缩短. 但是, 由于常规的电泳仪器即可操作台阶梯度, 而且能获得与 线形梯度相当的分离能力, 因此, 台阶梯度能够更便捷 地改善分离的效果.

在我们的前期工作中, 已经制备了多种动态磁涂覆 纳米固定相开管毛细管电色谱柱 ${ }^{[19,20]}$, 不同功能的磁性 纳米颗粒在外加磁铁的作用下, 吸附于毛细管的表面, 构建开管毛细管电色谱系统. 该方法制备简单, 仅通过 开/关磁场就能方便除去被污染的固定相, 快速实现色 谱柱的再生, 延长毛细管的使用寿命. 本文利用前期制 备的动态磁涂覆混合固定相(图 1)(离子交换/反相)开管 毛细管电色谱柱, 从理论上推导出等度洗脱和梯度洗脱

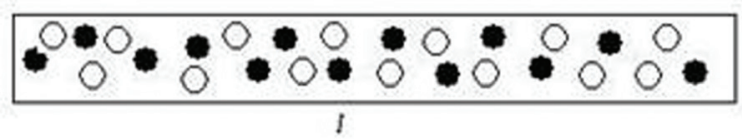

图 1 混合固定相示意图

Figure 1 Schematic diagram of mixed stationary phase
下芳香酸在该色谱柱中的输运方程. 进一步从实验上考 察两种固定相配比的改变对电渗流及等度洗脱下溶质 迁移的影响, 以及离子强度、有机调节剂浓度和 $\mathrm{pH}$ 值 等参数的改变对二元台阶梯度下溶质迁移的影响. 并以 水提天麻为样品对系统加以评价.

\section{2 结果与讨论}

\section{1 理论部分}

\section{1 .1 等度洗脱条件下溶质的保留特征}

混合固定相模式下, 溶质的保留特征可以用下式表 示

$$
t_{R}=t_{0}^{\text {iso }}(1+k)
$$

式中: $t_{0}^{i s o}$ 为溶质在等度洗脱混合固定相毛细管电色谱 中的死时间, $k$ 为溶质在混合固定相毛细管电色谱中的 容量因子.

假设体系中溶质的初始质量为 $m_{0}$, 则有

$$
m_{0}=m_{1}+m_{\mathrm{s} 1}+m_{\mathrm{s} 2}
$$

式中: $m_{1}$ 为溶质在流动相中的质量, $m_{\mathrm{s} 1}$ 为离子交换固定 相吸附的溶质质量, $m_{\mathrm{s} 2}$ 为反相固定相吸附的溶质质量.

在混合固定相毛细管电色谱中，体系中包含溶质的 三种形态, 其各自的摩尔分数分别为

$$
x_{1}=\frac{m_{\mathrm{s} 1}}{m_{0}}=\frac{\beta_{1} k_{1}}{1+\beta_{1} k_{1}+\beta_{2} k_{2}}
$$

式中: $x_{1}$ 为吸附在离子交换固定相上溶质的摩尔分数, $k_{1}$ 为在离子交换固定相和流动相间分配的溶质的容量因 子, $k_{2}$ 为在反相固定相和流动相间分配的溶质的容量因 子, $\beta_{1}$ 为流动相体积与离子交换固定相体积的比值, $\beta_{2}$ 为 流动相体积与反相固定相体积的比值.

$$
x_{2}=\frac{m_{\mathrm{s} 2}}{m_{0}}=\frac{\beta_{2} k_{2}}{1+\beta_{1} k_{1}+\beta_{2} k_{2}}
$$

式中: $x_{2}$ 为吸附在反相固定相上溶质的摩尔分数.

$$
x_{m}=\frac{m_{1}}{m_{0}}=\frac{1}{1+\beta_{1} k_{1}+\beta_{2} k_{2}}
$$

式中: $x_{m}$ 为流动相中溶质在初始质量 $m_{0}$ 中所占摩尔分 数.

根据分子独立运动原理 ${ }^{[21]}$, 溶质每一种形态的输 运过程均独立完成, 与其他形态的输运过程无关. 溶质 在电色谱过程中的宏观迁移速率等于其各种形态沿柱 轴向输运速率按摩尔分数的加权平均

$$
\mu=\sum_{i=1}^{n} \mu_{i} x_{i}
$$

式中: $\mu$ 为溶质的宏观迁移速率; $x_{i}$ 为其形态 $i$ 的摩尔分 数; $\mu_{i}$ 是指 $i$ 形态的溶质沿柱轴向的输运速率; $n$ 为其总 形态数.

对于被吸附在固定相中的溶质而言, 其迁移速率为 0 ; 而对于在流动相中的溶质而言, 其迁移速率为电泳 
流速率和电渗流速率的加和, 定义为 $\mu_{0}$.

结合式(3) (6), 溶质在等度洗脱混合固定相毛细 管电色谱中的迁移速率(单位: $\mathrm{cm} / \mathrm{s}$ )为

$$
\mu_{\text {iso }}=\frac{\mu_{0}}{1+\beta_{1} k_{1}+\beta_{2} k_{2}}
$$

从而, 溶质在等度洗脱混合固定相毛细管电色谱中 的迁移时间为

$$
t_{\text {iso }}=\frac{L}{\mu_{\text {iso }}}=t_{0}^{\text {iso }}\left(1+\beta_{1} k_{1}+\beta_{2} k_{2}\right)
$$

此外, 我们还考察了制备过程中 $\mathrm{Fe}_{3} \mathrm{O}_{4} @ \mathrm{SiO}_{2}-\mathrm{NH}_{2}$ 和 $\mathrm{Fe}_{3} \mathrm{O}_{4} @ \mathrm{SiO}_{2}-\mathrm{C}_{18}$ 悬浮液浓度对电渗流的影响, 结果表 明, 这一参数对电渗流影响较小, 当纳米颗粒浓度从 $1.0 \mathrm{mg} / \mathrm{mL}$ 增加到 $7.5 \mathrm{mg} / \mathrm{mL}$, 死时间变化幅度仅为 0.5 $\min$.

\section{1 .2 二元台阶梯度洗脱条件下溶质的保留特征}

将混合固定相作为一种固定相考虑, 处理过程和单 独一种固定相时相同.

溶质在完成第一个台阶梯度时间内迁移的距离 $l_{1}$ 为

$l_{1}=\mu_{\mathrm{gra}} t_{1} /\left(1+\beta_{b 1} k_{11}\right)$

式中: $t_{1}$ 为溶质完成第一个台阶梯度洗脱所需要的时间; $\beta_{b 1}$ 为第一种流动相体积与混和固定相体积的比值; $k_{11}$ 为在混合固定相和第一种流动相中分配的溶质的容量 因子; $\mu_{\mathrm{gra}}$ 为溶质的表观迁移速率.

剩下的距离在第二种流动相驱动下完成, 所需的时 间 $t_{2}$ 为

$$
t_{2}=\frac{t_{0}^{\mathrm{gra}}-t_{1} /\left(1+\beta_{b 1} k_{11}\right)}{1 /\left(1+\beta_{b 2} k_{12}\right)}
$$

式中: $t_{0}^{\mathrm{gra}}$ 为溶质在二元台阶梯度洗脱混合固定相毛细 管电色谱中的死时间; $\beta_{b 2}$ 为第二种流动相体积与混合固 定相体积的比值; $k_{12}$ 为在混合固定相和第二种流动相间 分配的溶质的容量因子.

从而, 溶质总的迁移时间为

$$
t_{\text {gra }}=t_{1}+\frac{t_{0}^{\mathrm{gra}}-t_{1} /\left(1+\beta_{b 1} k_{11}\right)}{1 /\left(1+\beta_{b 2} k_{12}\right)}
$$

式(8)和式(11)分别描述了溶质在等度洗脱和二元台阶 梯度洗脱条件下的迁移时间的变化规律. 两种固定相的 配比, 两步台阶梯度缓冲液的性质等都将影响溶质的迁 移.

\section{2 电渗流的变化}

在离子交换色谱中, WAX 是较常用的色谱固定相, 其表面氨基解离后具有较强的亲水性; 在反相色谱中, ODS 是最常用的具有疏水能力的反相色谱固定相. 采 用 WAX 与 ODS 固定相构建混合固定相毛细管色谱柱 具有代表性. 在我们前期工作中 ${ }^{[23]}$, 在不考虑这两种固

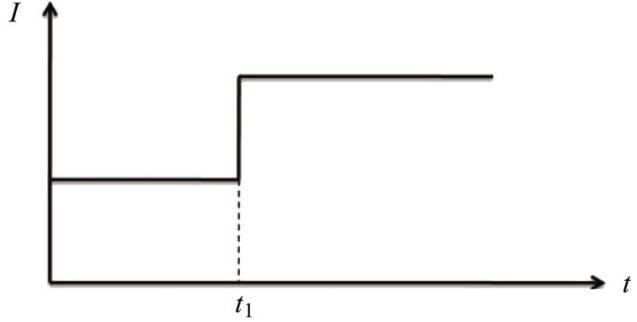

图 2 二元台阶梯度示意图

Figure 2 Schematic diagram of double stepwise gradient $t_{1}$ is the elution time of solute in the first stepwise elution of double stepwise gradient elution

定相之间的交互影响，且认为两者密度相同的情况下， 推导得出电渗流与混和固定相配比间关系式，也即随着 阴离子交换固定相配比的增加，正向电渗流逐渐变小. 在 $\mathrm{Fe}_{3} \mathrm{O}_{4} @ \mathrm{SiO}_{2}-\mathrm{NH}_{2}$ 所占比例在 55.5\% 81.2\%(质量比) 间变化时, 我们得到了相似的结果, 结果如图 3 所示.

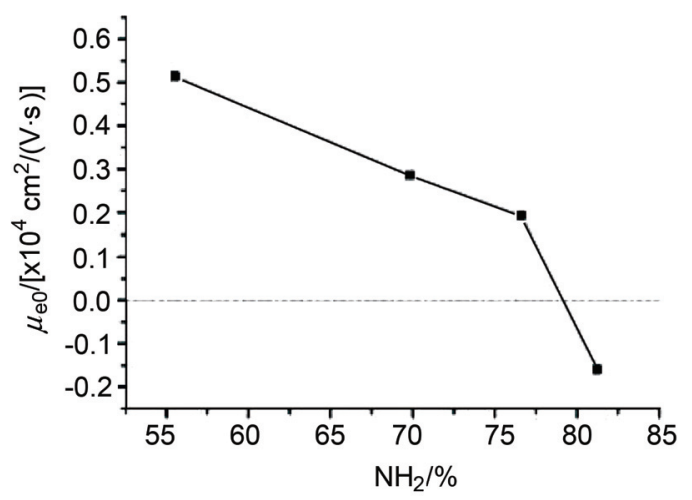

图 3 混合固定相不同配比对电渗流淌度的影响

Figure 3 Effect of different ratio of mixed stationary phase on EOF Experimental conditions: buffer, $50 \% \mathrm{ACN}, 10 \mathrm{mmol} / \mathrm{L} \mathrm{NaH} \mathrm{PO}_{4}(\mathrm{pH} 6.2$ ); Injection voltage, $-3 \mathrm{kV}, 3 \mathrm{~s}$

可以看出, 当填充入色谱柱的 $\mathrm{Fe}_{3} \mathrm{O}_{4} @ \mathrm{SiO}_{2}-\mathrm{NH}_{2}$ 和 $\mathrm{Fe}_{3} \mathrm{O}_{4} @ \mathrm{SiO}_{2}-\mathrm{C}_{18}$ 质量比发生改变时, 由于产生电渗流的 基团的种类和数量发生改变, 色谱柱的电渗流的大小也 相应的发生变化，甚至导致最终方向发生改变 $\left(\mathrm{Fe}_{3} \mathrm{O}_{4} @ \mathrm{SiO}_{2}-\mathrm{NH}_{2} \%=81.2 \%\right)$. 由于制备的开管柱柱床 的带电情况主要取决于氨基的数量、毛细管壁和固定相 表面残留的硅羟基. 正向电渗流主要由毛细管管壁残留 的硅羟基和 $\mathrm{Fe}_{3} \mathrm{O}_{4} @ \mathrm{SiO}_{2}$ 表面残留的硅羟基产生，而反 向电渗流主要由 $\mathrm{Fe}_{3} \mathrm{O}_{4} @ \mathrm{SiO}_{2}-\mathrm{NH}_{2}$ 固定相表面解离的氨 基产生. 三者的综合作用决定了电渗流的方向和大小. 实验发现，制备的开管柱在 $\mathrm{Fe}_{3} \mathrm{O}_{4} @ \mathrm{SiO}_{2}-\mathrm{NH}_{2} / \mathrm{Fe}_{3} \mathrm{O}_{4} @$ $\mathrm{SiO}_{2}-\mathrm{C}_{18}=1 \sim 3$ 时, 具有从阳极流向阴极的电渗流. 在 该条件下，毛细管壁和固定相表面残留的硅羟基带有较 多的负电荷，随着氨基比例的增加，电渗流降低. 当 $\mathrm{Fe}_{3} \mathrm{O}_{4} @ \mathrm{SiO}_{2}-\mathrm{NH}_{2} / \mathrm{Fe}_{3} \mathrm{O}_{4} @ \mathrm{SiO}_{2}-\mathrm{C}_{18}=4$ 时，质子化的氨基 使柱床表面带有较多的正电荷，电渗流方向发生转向， 
从阴极到阳极. 由于受到磁性纳米固定相改性重复性、 柱制备条件的限制，不同批次制备的 OT-CEC 柱间存在 一定误差(保留时间相对标准偏差 $>5 \%$ ), 但是混合固定 相中氨基比例增加, 最终一定会导致电渗流方向和大小 都发生变化

\section{3 等度洗脱条件下溶质在不同配比混合固定相毛细 管色谱柱中的输运特征}

从式(8)可以看出, 随着固定相中 $\mathrm{Fe}_{3} \mathrm{O}_{4} @ \mathrm{SiO}_{2}-\mathrm{NH}_{2}$ 和 $\mathrm{Fe}_{3} \mathrm{O}_{4} @ \mathrm{SiO}_{2}-\mathrm{C}_{18}$ 配比 $\eta$ 的改变, $\beta$ 也随之发生变化.

进一步地, 假设 $\beta_{1}=a_{1} \eta+b_{1}, \beta_{2}=a_{2} \eta+b_{2}, b$ 为校正 因子.

其中: $b_{1}$ 校正 $\mathrm{Fe}_{3} \mathrm{O}_{4} @ \mathrm{SiO}_{2}-\mathrm{NH}_{2}$ 表面聚合物材料的 疏水性等因素的影响, $b_{2}$ 校正 $\mathrm{Fe}_{3} \mathrm{O}_{4} @ \mathrm{SiO}_{2}-\mathrm{C}_{18}$ 表面残留 的硅差基等因素的影响.

结合式(8), 可以得到

$$
k=\frac{t_{R}-t_{0}}{t_{0}}=A \eta+B
$$

式中: $A=k_{1} a_{1}+k_{2} a_{2}, B=k_{1} b_{1}+k_{2} b_{2}$.

从式(12)可以看出, 溶质在混合固定相毛细管电色 谱中, 容量因子与 $\mathrm{Fe}_{3} \mathrm{O}_{4} @ \mathrm{SiO}_{2}-\mathrm{NH}_{2}$ 和 $\mathrm{Fe}_{3} \mathrm{O}_{4} @ \mathrm{SiO}_{2}-\mathrm{C}_{18}$ 的配比之间具有线性关系.

在其他条件不变的情况下, 以有机酸为样品, 通过 改变 $\mathrm{Fe}_{3} \mathrm{O}_{4} @ \mathrm{SiO}_{2}-\mathrm{NH}_{2}$ 和 $\mathrm{Fe}_{3} \mathrm{O}_{4} @ \mathrm{SiO}_{2}-\mathrm{C}_{18}$ 的质量比, 考 察了配比与容量因子之间的关系, 结果如图 4 所示.

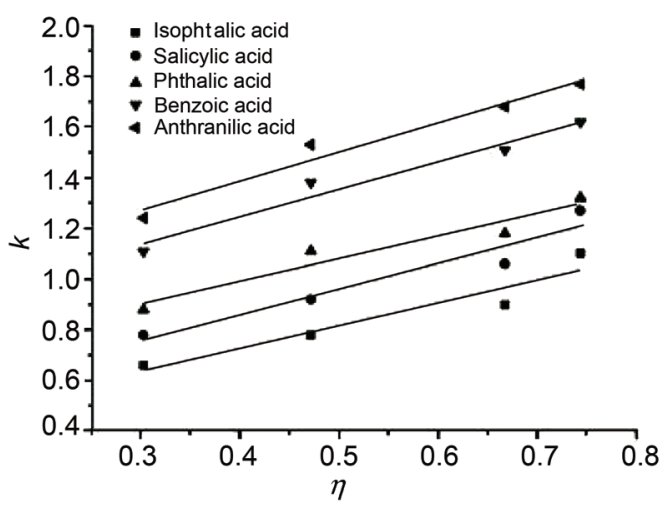

图 4 配比 $\eta$ 与容量因子 $k$ 关系图

Figure 4 Relationship diagram of $\eta$ and $k$

溶质的容量因子由式(1)计算得到, 其中, $t_{0}$ 为溶剂 峰的保留时间. 从图 4 可以看出, 随着混合固定相中氨 基配比的增加, 溶质的容量因子也随之增加. 在 $\mathrm{pH} 6$ 的 条件下, 五种有机酸主要以负电形态存在. 溶质的迁移 不仅依靠电泳机理, 还存在着色谱机理. 有机酸在系统 中不仅随电渗流驱动着流动相前进, 此外还有其自身的 泳动. 带电基团可以与氨基固定相发生离子交换相互作 用，同时分子中疏水基团也与 $\mathrm{C}_{18}$ 固定相发生相互作用. 随着氨基比例的增加, 柱床表面带有更多的正电荷, 正 向电渗流降低, 同时, 溶质与其之间发生离子交换相互
作用的次数增加, 保留增加, 导致出峰时间延长. 而随 着 $\mathrm{C}_{18}$ 比例的降低, 溶质与反相固定相之间发生疏水相 互作用的次数降低，使得保留减弱. 几者的综合作用显 示出如图 4 所示的结果, 可以看出, 随着氨基比例的增 加, 在有机酸的分离过程中, 离子交换作用与电泳行为 相比起到了更主要的作用. 进一步地, 根据色谱数据, 经式(12)拟合后, 得到的结果如表 1 所示.

表 1 拟合方程及相关系数 ${ }^{a}$

Table 1 Fitting equations and correlation coefficients

\begin{tabular}{llc}
\hline Organic acid & Fitting equation & Correlation coefficient $\left(R^{2}\right)$ \\
\hline Isophtalic acid & $k=0.90 \eta+0.37$ & 0.9166 \\
Salicylic acid & $k=1.00 \eta+0.46$ & 0.9331 \\
Phthalic acid & $k=0.90 \eta+0.63$ & 0.9313 \\
Benzoic acid & $k=1.08 \eta+0.82$ & 0.9643 \\
Anthranilic acid & $k=1.15 \eta+0.93$ & 0.9647
\end{tabular}

${ }^{a}$ Experimental conditions: buffer, $50 \% \mathrm{ACN}, 10 \mathrm{mmol} / \mathrm{L} \mathrm{NaH}_{2} \mathrm{PO}_{4}(\mathrm{pH} 6.2)$; Injection voltage, $-2 \mathrm{kV}, 5 \mathrm{~s}$.

可以看出，有机酸的容量因子与固定相配比经拟合 后，线性良好，相关系数 $R^{2}$ 大于 0.9166 . 式(12)能够很 好地描述溶质在混合固定相等度洗脱中固定相配比与 保留因子之间的规律.

\section{4 二元台阶梯度条件下溶质在混合固定相毛细管色 谱柱中的保留特征}

在毛细管电色谱中, 流动相种类改变后, 由于缓冲 液浓度、有机调节剂黏度等性质的改变，导致流动相的 流速发生改变, 溶质的迁移时间也相应发生改变. 为了 考察溶质在毛细管电色谱二元台阶梯度洗脱中的输运 规律, 式(11)进一步改写成

$$
\begin{aligned}
& t_{\mathrm{gra}}=t_{1}+t_{0}^{\mathrm{gra}}\left(1+\beta_{b 2} k_{12}\right)-t_{1} \frac{t_{0}^{\mathrm{gra}}\left(1+\beta_{b 2} k_{12}\right)}{t_{0}^{\mathrm{gra}}\left(1+\beta_{b 1} k_{11}\right)} \\
& \text { 从而 } \\
& t_{\mathrm{gra}}=t_{1}+t_{R 2}-t_{1} \frac{t_{R 2}}{t_{R 1}}
\end{aligned}
$$

式中: $t_{1}$ 是二元台阶梯度洗脱条件下施加第一种流动相 的运行时间, $t_{\mathrm{R} 1}$ 是溶质在第一种流动相等度洗脱条件下 的洗脱时间, $t_{\mathrm{R} 2}$ 是溶质在第二种流动相等度洗脱条件下 的洗脱时间.

式(14)可以进一步说明溶质的输运特征, 溶质在二 元台阶梯度洗脱条件下的迁移时间可以通过施加第一 种流动相的运行时间、其在第一种和第二种流动相等度 洗脱条件下的洗脱时间加以预测.

在实际台阶梯度毛细管电色谱操作中, 由于溶剂切 换，需考虑梯度延迟时间. 式(14)进一步改写为:

$$
t_{\mathrm{gra}}=\left(t_{1}+\alpha\right)+t_{R 2}-\left(t_{1}+\alpha\right) \frac{t_{R 2}}{t_{R 1}}
$$

式中: $\alpha$ 是梯度延迟时间校正因子，它可以表示为 


$$
\alpha=\frac{\frac{l}{t_{\mathrm{R} 1}} t_{1}}{\frac{l}{t_{02}}}=\frac{t_{1} t_{02}}{t_{\mathrm{R} 1}}
$$

式中: $l$ 是毛细管的有效长度, $t_{02}$ 是溶质在第二种流动相 等度洗脱下的死时间.

为了验证理论的可靠性, 以邻氨基苯甲酸和对氨基 苯甲酸为样品分别在等度洗脱和二元台阶梯度洗脱条 件下考察其迁移规律. 实验中设定 $t_{1}=6.5 \mathrm{~min}$. 进一步 根据式(15)和式(16) 计算出溶质分别在磷酸缓冲液浓 度、乙腈浓度和 $\mathrm{pH}$ 值二元台阶梯度洗脱条件下混合固 定相毛细管电色谱柱中的理论迁移时间, 与实验结果相 比较, 计算出相对误差, 结果如表 2-1、表 2-2 和表 2-3 所示.

溶质在二元台阶梯度洗脱条件下混合固定相毛细 管电色谱柱中的实际迁移时间由其在第一种流动相和 第二种流动相洗脱时间的加和得到(其中, 溶质在第一 种流动相中的洗脱时间 $t_{1}=6.5 \mathrm{~min}$ ). 从表 $2-1$ 、表 $2-2$ 和表 2-3 可以看出, 溶质在经磷酸缓冲液浓度二元台阶 梯度洗脱后, 保留时间的理论与实际值相对误差小于 $8.0 \%$; 溶质在经有机调节剂浓度二元台阶梯度洗脱后, 保留时间的理论与实际值相对误差小于 $10 \%$; 溶质在经
$\mathrm{pH}$ 值二元台阶梯度洗脱后，保留时间的理论与实际值 相对误差小于 $5.8 \%$. 由于在动态磁涂覆混合固定相毛 细管电色谱柱的制备和运行过程中, 多种制备和分离参 数如悬浮液浓度、流速等都影响着色谱柱的重复性和稳 定性，造成保留时间测定误差. 因此尽管理论值与实际 值间误差较大，该理论表达式仍然能够在一定程度上描 述溶质在毛细管电色谱二元台阶梯度洗脱中的输运规律.

\section{5 混合固定相毛细管色谱柱上芳香酸的分离}

优化分离条件后，芳香酸在动态磁涂覆混合纳米固 定相开管毛细管柱中分离谱图如图 5(a)所示. 不同纳米 颗粒涂覆下五种有机酸均获得基线分离, 但因固定相性 质不同，分离后效果也不同. 混和固定相涂覆下，苯甲 酸柱效达 480000 塔板数 $/ \mathrm{m}$, 连续三次进样后的相对标 准偏差小于 $1.65 \%$. 与单一固定相(阴离子交换或反相) 比，分离柱效大大加强(表 3).

\section{6 应用}

天麻是兰科植物天麻的干燥块茎，在临床上主要用 于治疗血管神经性头痛和神经衰弱等疾病. 在一维分离 模式下不能得到理想的分离结果，优化条件下，采用制 备的动态磁涂覆混合固定相开管毛细管电色谱柱对水 提天麻进行分离，分离谱图如图 6 所示.

表 2-1 二元台阶梯度(磷酸缓冲液浓度)电色谱分离中有机酸保留时间(min)的预测 ${ }^{a}$

Table 2-1 Prediction of retention time (min) of solute in double stepwise gradient (phosphate buffer concentration) CEC separation

\begin{tabular}{|c|c|c|c|c|c|c|c|c|c|}
\hline & \multicolumn{3}{|c|}{$5 \rightarrow 10 \mathrm{mmol} / \mathrm{L}$} & \multicolumn{3}{|c|}{$10 \rightarrow 15 \mathrm{mmol} / \mathrm{L}$} & \multicolumn{3}{|c|}{$15 \rightarrow 20 \mathrm{mmol} / \mathrm{L}$} \\
\hline & Exp. & Theo. & $\%$ & Exp. & Theo. & $\%$ & Exp. & Theo. & $\%$ \\
\hline Anthranilic acid & 7.24 & 7.20 & -0.49 & 7.29 & 7.38 & 1.2 & 7.30 & 7.37 & 0.95 \\
\hline \multirow[t]{3}{*}{$p$-Aminobenzoic acid } & 8.87 & 9.12 & 2.8 & 8.99 & 9.48 & 5.2 & 9.23 & 9.57 & 3.5 \\
\hline & \multicolumn{3}{|c|}{$20 \rightarrow 25 \mathrm{mmol} / \mathrm{L}$} & \multicolumn{3}{|c|}{$25 \rightarrow 30 \mathrm{mmol} / \mathrm{L}$} & & & \\
\hline & Exp. & Theo. & $\%$ & Exp. & Theo. & $\%$ & & & \\
\hline Anthranilic acid & 7.28 & 7.54 & 3.4 & 7.27 & 7.55 & 3.7 & & & \\
\hline$p$-Aminobenzoic acid & 9.29 & 9.75 & 4.8 & 9.35 & 10.11 & 7.5 & & & \\
\hline
\end{tabular}

${ }^{a}$ Experimental conditions: $\mathrm{NH}_{2} / \mathrm{C}_{18}=2$; buffer, $50 \% \mathrm{ACN}$, phosphate buffer with $\mathrm{pH} 6.2$; Injection voltage, $-1 \mathrm{kV}, 2 \mathrm{~s} ; 5 \mathrm{mmol} / \mathrm{L}$ phosphate buffer $\rightarrow 10 \mathrm{mmol} / \mathrm{L}$ phosphate buffer, $5 \mathrm{mmol} / \mathrm{L}$ phosphate buffer was the first stepwise elution mobile phase in the double stepwise gradient elution, $10 \mathrm{mmol} / \mathrm{L}$ phosphate buffer was the second stepwise elution mobile phase in the double stepwise gradient elution, and so on.

表 2-2 二元台阶梯度(有机调节剂浓度)电色谱分离中有机酸保留时间(min)的预测 ${ }^{a}$

Table 2-2 Prediction of retention time (min) of solute in double stepwise gradient (organic modifier concentration) CEC separation

\begin{tabular}{|c|c|c|c|c|c|c|c|c|c|}
\hline & \multicolumn{3}{|c|}{$40 \% \rightarrow 45 \%$} & \multicolumn{3}{|c|}{$45 \% \rightarrow 50 \%$} & \multicolumn{3}{|c|}{$50 \% \rightarrow 55 \%$} \\
\hline & Exp. & Theo. & $\%$ & Exp. & Theo. & $\%$ & Exp. & Theo. & $\%$ \\
\hline Anthranilic acid & 7.13 & 7.39 & 3.5 & 6.74 & 6.67 & -0.99 & 6.98 & 7.17 & 2.7 \\
\hline \multirow[t]{3}{*}{$p$-Aminobenzoic acid } & 8.42 & 8.69 & 3.2 & 8.17 & 8.35 & 2.13 & 8.83 & 9.30 & 5.1 \\
\hline & \multicolumn{3}{|c|}{$55 \% \rightarrow 60 \%$} & \multicolumn{3}{|c|}{$60 \% \rightarrow 65 \%$} & & & \\
\hline & Exp. & Theo. & $\%$ & Exp. & Theo. & $\%$ & & & \\
\hline Anthranilic acid & 7.69 & 7.39 & -4.1 & 7.72 & 7.99 & 3.3 & & & \\
\hline$p$-Aminobenzoic acid & 9.90 & 10.02 & 1.2 & 10.66 & 11.82 & 9.8 & & & \\
\hline
\end{tabular}

${ }^{a}$ Experimental conditions: $\mathrm{NH}_{2} / \mathrm{C}_{18}=2$; buffer, $10 \mathrm{mmol} / \mathrm{L}$ phosphate buffer with $\mathrm{pH} 6.2 ;$ Injection voltage, $-1 \mathrm{kV}, 2 \mathrm{~s} ; 40 \% \mathrm{ACN} \rightarrow 45 \% \mathrm{ACN}, 40 \% \mathrm{ACN}$ was the first stepwise elution mobile phase in the double stepwise gradient elution, $45 \% \mathrm{ACN}$ was the second stepwise elution mobile phase in the double stepwise gradient elution, and so on. 
表 2-3 二元台阶梯度( $\mathrm{pH}$ 值)电色谱分离中有机酸保留时间(min)的预测 ${ }^{a}$

Table 2-3 Prediction of retention time ( $\mathrm{min}$ ) of solute in double stepwise gradient ( $\mathrm{pH}$ value) CEC separation

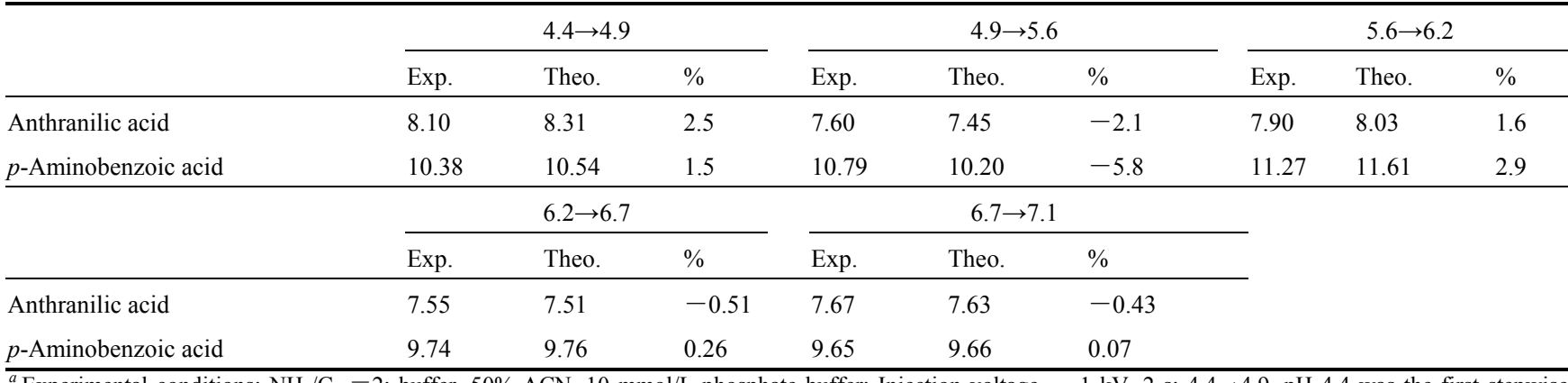

${ }^{a}$ Experimental conditions: $\mathrm{NH}_{2} / \mathrm{C}_{18}=2$; buffer, $50 \% \mathrm{ACN}, 10 \mathrm{mmol} / \mathrm{L}$ phosphate buffer; Injection voltage, $-1 \mathrm{kV}, 2 \mathrm{~s} ; 4.4 \rightarrow 4.9$, pH $4.4 \mathrm{kas}$ the first stepwise elution mobile phase in the double stepwise gradient elution, $\mathrm{pH} 4.9$ was the second stepwise elution mobile phase in the double stepwise gradient elution, and so on.

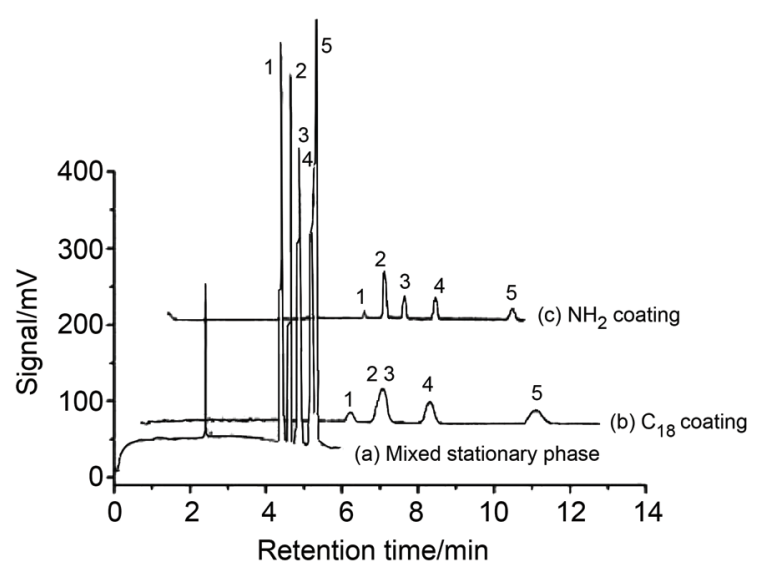

图 5 动态磁涂覆纳米固定相分离电色谱图

Figure 5 The separation electrochromatogram of nanoparticle coating Experimental conditions: (a) mixed stationary phase coating, (b) $\mathrm{Fe}_{3} \mathrm{O}_{4} @ \mathrm{SiO}_{2}-\mathrm{C}_{18}$ coating, (c) $\mathrm{Fe}_{3} \mathrm{O}_{4} @ \mathrm{SiO}_{2}-\mathrm{NH}_{2}$ coating. Capillary length, $11 / 22 / 30 \mathrm{~cm}$. Separation voltage: $-267 \mathrm{~V} / \mathrm{cm}$. Temperature: room temperature. UV-Vis detector wave length: $214 \mathrm{~nm}$. Data collection frequency: $16 \mathrm{~Hz}$. Mobile phase: $50 \% \mathrm{ACN}, 20 \mathrm{mmol} / \mathrm{L} \mathrm{NaH}_{2} \mathrm{PO}_{4}(\mathrm{pH}=7.0)$. Injection voltage: $-6 \mathrm{kV}, 10 \mathrm{~s}$. Organic acids: 1 , isophtalic acid; 2 , salicylic acid; 3 , phthalic acid; 4 , benzoic acid; 5 , anthranilic acid.

制备的毛细管柱具有正向电渗流，该实验条件下其 大小为 $1.82 \times 10^{-4} \mathrm{~cm}^{2} /(\mathrm{V} \cdot \mathrm{s})$. 采用该色谱柱, 天麻中主 要成分在 $40 \mathrm{~min}$ 之内被洗脱, 其中香草醇、柠檬酸和天 麻素根据标准品保留时间定性. 同时, 柠檬酸经磷酸缓 冲液浓度二元台阶梯度洗脱后, 采用式(15)和式(16)预 测，其理论保留时间与实际保留时间的相对误差小于 $11 \%(15 \mathrm{mmol} / \mathrm{L}$ 磷酸盐等度洗脱时, 其出峰时间为 $10.06 \mathrm{~min} ; 20 \mathrm{mmol} / \mathrm{L}$ 磷酸盐等度洗脱时, 其出峰时间 为 $6.47 \mathrm{~min}$; 采用台阶梯度洗脱, 柠檬酸在第一种流动 相 $15 \mathrm{mmol} / \mathrm{L}$ 磷酸盐中的迁移时间为 $6.00 \mathrm{~min}$, 其在第 二种流动相 $20 \mathrm{mmol} / \mathrm{L}$ 中的迁移时间为 $2.73 \mathrm{~min}$ ). 考虑 到较小的电渗流, 多重电极设置确保天麻中带负电、中 性和带正电组分均能被洗脱. 当电极设置柱进口为阳极 时, 带正电的天麻素和香草醇在电渗流和电泳流的共同 驱动下迁移出色谱柱; 当电极设置柱进口为阴极时, 带 负电的柠檬酸电泳速度较快, 大于电渗流, 它的迁移主
表 3 分离参数数据

Table 3 Data of separation parameters

\begin{tabular}{lccccccc}
\hline & \multicolumn{4}{c}{ Resolution $^{a}$} & & $N$ (plates $/ \mathrm{m}$ ) & RSD $\%$ \\
\cline { 2 - 5 } & $R_{1}$ & $R_{2}$ & $R_{3}$ & $R_{4}$ & & \\
\hline IEC & 3.97 & 3.70 & 5.48 & 10.13 & & 300000 for phthalic acid & 2.73 \\
RP & 2.76 & 0 & 2.73 & 5.33 & & 250000 for isophtalic acid & 0.93 \\
Isocratic & 2.48 & 2.17 & 3.02 & 1.32 & & 480000 for benzoic acid & 1.65 \\
elution & & & & & & \\
\hline
\end{tabular}

${ }^{a}$ Where $R_{1}$ is the resolution of isophtalic acid and salicylic acid; $R_{2}$ is the resolution of salicylic acid and phthalic acid; $R_{3}$ is the resolution of phthalic acid and benzoic acid; $R_{4}$ is the resolution of benzoic acid and anthranilic acid. (a)

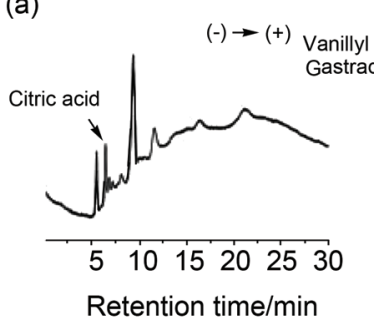

(b) $\quad(+) \rightarrow(-)$

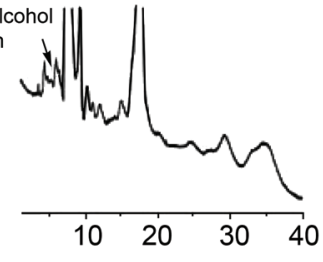

Retention time/min
图 6 水提天麻分离谱图

Figure 6 Separation electrochromatogram of rhizoma gastrodiae Experimental conditions: (a) electrode from cathode to anode, (b) electrode from anode to cathode; buffer, $50 \% \mathrm{ACN}, 20 \mathrm{mmol} / \mathrm{L} \mathrm{Na}_{2} \mathrm{HPO}_{4}(\mathrm{pH} 7.6$ ); Injection voltage, (a) $(-8 \mathrm{kV}, 15 \mathrm{~s})$, (b) $(+6 \mathrm{kV}, 6 \mathrm{~s})$.

要依靠电泳流. 可以看出, 这种新型动态磁涂覆混合纳 米固定相毛细管电色谱柱满足对复杂样品分离分析方 法发展的需要.

\section{3 结论}

本文从理论上推导出等度洗脱和梯度洗脱下芳香 酸在动态磁涂覆混合纳米固定相毛细管电色谱柱中的 输运特征, 说明两种固定相的配比等参数都影响溶质的 迁移. 同时, 溶质在等度洗脱下的保留时间能够很好地 预测其在二元台阶梯度下的保留时间. 结果表明, 等度 洗脱下，芳香酸在该色谱柱中容量因子与混合固定相配 比之间具有良好的线性关系. 溶质在经缓冲液浓度、有 机调节剂浓度和 $\mathrm{pH}$ 值台阶梯度洗脱后, 其实际测量保 留时间与理论值基本相符. 在优化的色谱条件下，对复 
杂天然产物水提天麻进行了分离, 较大的峰容量也表明 该色谱柱适用于复杂天然产物的分离.

\section{4 实验部分}

\section{1 磁性纳米材料的功能化}

核壳磁性纳米颗粒采用文献[19]的方法制备, 制备 完成后, 得到粒径约为 $100 \mathrm{~nm}$ 的 $\mathrm{Fe}_{3} \mathrm{O}_{4} @ \mathrm{SiO}_{2}$. 纳米材 料的功能化按照文献 [19，22]的方法制备(具体制备过程 见补充材料), 制备完成后, 分别得到具有离子交换功 能的固定相 $\mathrm{Fe}_{3} \mathrm{O}_{4} @ \mathrm{SiO}_{2}-\mathrm{NH}_{2}$ 和反相功能的固定相 $\mathrm{Fe}_{3} \mathrm{O}_{4} @ \mathrm{SiO}_{2}-\mathrm{C}_{18}$.

\section{2 动态磁涂覆混合固定相开管毛细管电色谱柱的制 备}

采用文献[19]方法制备动态磁涂覆混合固定相开管 毛细管电色谱柱: 在毛细管上对夹 30 对同心圆磁铁. 在 $12.50 \mu \mathrm{L} / \mathrm{min}$ 的流速下将总浓度为 $5.0 \mathrm{mg} / \mathrm{mL}$ 不同比例 的 $\mathrm{Fe}_{3} \mathrm{O}_{4} @ \mathrm{SiO}_{2}-\mathrm{NH}_{2}$ 和 $\mathrm{Fe}_{3} \mathrm{O}_{4} @ \mathrm{SiO}_{2}-\mathrm{C}_{18}$ 纳米颗粒悬浮液 通入毛细管中, 利用磁铁产生的磁场和与毛细管壁产生 的摩擦力, 使其均匀、有序地沿着毛细管壁排列, 通过 从磁铁对的缝隙观察填料的装填进度. 在相同的流速 下，用微流注射泵将磷酸盐缓冲液推入毛细管中, 洗去 未被固定的纳米颗粒. 制备完成后, 毛细管总长为 30 $\mathrm{cm}$, 有效长度为 $22 \mathrm{~cm}$, 其中, 第 1 对磁铁与毛细管入 口之间距离为 $3.5 \mathrm{~cm}$, 第 30 对磁铁与检测窗口之间距 离为 $5.5 \mathrm{~cm}$.

\section{3 电色谱条件}

分离场强为 $267 \mathrm{~V} / \mathrm{cm}$, 紫外检测波长为 $214 \mathrm{~nm}$, 数 据采集频率为 $16 \mathrm{~Hz}$ ，分离温度为室温.

\section{References}

[1] Huber, C. G.; Choudhary, G.; Horváth, C. Anal. Chem. 1997, 69, 4429.

[2] Ericson, C.; Hjerntén, S. Anal. Chem. 1999, 71, 1621.

[3] Lister, A. S.; Rimmer, C. A.; Dorsey, J. G. J. Chromatogr. A 1998, $828,105$.

[4] Euerby, M. R.; Johnson, C. M.; Cikalo, M.; Bartle, K. D. Chromatographia 1998, 47, 135

[5] Djordjevic, N. M.; Fitzpartrick, F.; Houdiere, F.; Lerch, G.; Rozing, G. J. Chromatogr. A 2000, 887, 245.

[6] Sepaniak, M. J.; Swaile, D.; Powell, A. G. J. Chromatogr. A 1989, $480,185$.

[7] Tsuda, T. Anal. Chem. 1992, 64, 386.

[8] Yan, C.; Dadoo, R.; Zare, R. N. Anal. Chem. 1996, 68, 2726.

[9] Behnke, B.; Bayer, E. J. Chromatogr. A 1994, 680, 93.

[10] Nakashima, R.; Kitagawa, S.; Yoshida, T.; Tsuda, T. J. Chromatogr. A 2004, 1044, 305.

[11] Kahle, V.; Vázlerová, M.; Welsch, T. J. Chromatogr. A 2003, 990 3

[12] Kahle, V.; Kost' ál, V.; Zeisbergerová, M. J. Chromatogr. A 2004, 1044, 259.

[13] Euerby, M. R.; Gilligan, D.; Johnson, C. M.; Bartle, K. D. Analyst 1997, 122, 1087.

[14] Sudor, J.; Stránský, Z.; Pospíchal, J.; Deml, M.; Boček, D. P. Electrophoresis 1989, 10, 802.

[15] Boček, P.; Deml, M.; Pospíchal, J.; Sudor, J. J. Chromatogr. A 1989, $470,309$.

[16] Balchunas, A. T.; Sepaniak, M. J. Anal. Chem. 1988, 60, 617.

[17] Zhang, Y. K.; Shi, W.; Zhang, L. H.; Zou, H. F. J. Chromatogr. A 1998, 802, 59.

[18] Zhang, W. B.; Zhang, L. H.; Ping, G. C.; Zhang, Y. K.; Kettrup, A. J. Chromatogr. A 2001, 922, 277.

[19] Zhu, Y. X.; Zhou, C. R.; Qin, S. S.; Ren, Z. Y.; Zhang, L. Y.; Fu, H. G.; Zhang, W. B. Electrophoresis 2012, 33, 340.

[20] Zhu, Y. X.; Zhang, L. Y.; Qian, J. H.; Zhang, W. B. Talanta 2013, 104, 174.

[21] Zhang, W. B. Theoretic Bases of Capillary Electrochromatography, Science Press, Beijing, 2006, p. 97.

[22] Tian, R. J.; Sun, J. M.; Zhang, H.; Ye, M. L.; Xie, C. H.; Dong, J.; Hu, J. W.; Ma, D.; Bao, X. H.; Zou, H. F. Electrophoresis 2006, 27, 742 .

[23] Qin, S. S.; Zhou, C. R.; Zhu, Y. X.; Ren, Z. Y.; Zhang, L. Y.; Fu, H. G.; Zhang, W. B. Chin. J. Chromatogr. 2011, 29, 942. 